\title{
Tersoff Selected as 1996 MRS Medalist
}

Jerry Tersoff of the IBM T.J. Watson Research Center has been selected to receive the 1996 MRS Medal, an honor that recognizes recent achievements or discoveries which are expected to have a major impact on the progress of any materials-related field.

Tersoff was cited "for his seminal contributions to the theory of strain relaxation in thin films." The relaxation of strain in thin films has long been a key problem in materials science.

Tersoff has revolutionized the understanding of key aspects of strain relaxation. He showed that strain-induced roughening differs from what had been believed based on continuum models. In particular, he confirmed that strained layers are metastable rather than unstable. This work provided a new understanding of methods for suppressing roughness during strained-layer growth.

Relaxation by roughening and introduction of dislocations had been treated as unrelated problems, but Tersoff tied them together, showing a kinetic competition between roughening and direct introduction of dislocations. This competition provides the key to controlling film quality.

In addition, he has made numerous contributions to the understanding of other aspects of strain relaxation, such as the dis-

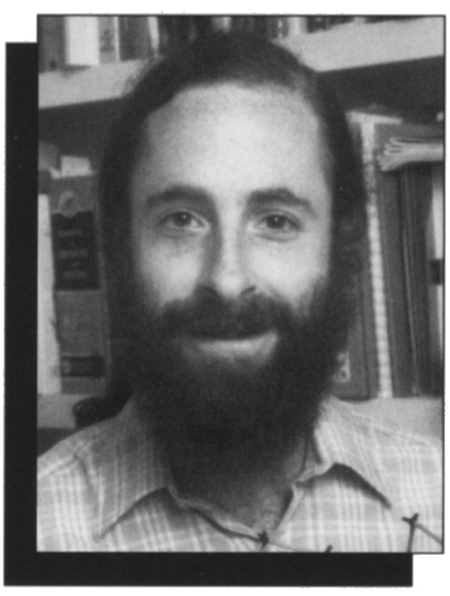

tribution of dislocations in graded layers, measurement of nucleation barriers for dislocations, and the origin of sessile Lomer dislocations. He showed that elastic strain relaxation is the driving mechanism for formation of a new two-dimensional alloy phase observed in metal heteroepitaxy, and most recently, demonstrated how elastic strain relaxation can be used to aid nanofabrication.

Until Tersoff's work, strain-induced roughening was treated entirely from a continuum viewpoint, but he noted that real semiconductor growth involves dis- crete facets or steps on low-index surfaces. By treating growth from this more realistic standpoint, he showed that the morphological evolution is entirely different from what had been believed.

Most recently, he has introduced a twist in the theoretical understanding of elastic relaxation of strained layers, and has shown how strain-induced roughening drives the self-organization of quantumdot superlattices.

Tersoff earned a BA degree in physics from Swarthmore College in 1977, and a $\mathrm{PhD}$ degree in physics in 1982 from the University of California-Berkeley. He began his career as a post-doc at AT\&T Bell Laboratories, and joined the IBM T.J. Watson Research Center in 1984. In 1988, he received the Peter Mark Memorial Award from the American Vacuum Society, and was elected a fellow of the American Physical Society in 1994.

In addition, he received an IBM Outstanding Technical Achievement Award in 1992. Tersoff is the author of more than 60 scientific papers, and has delivered almost 70 invited conference talks.

Tersoff will give his Medalist Award talk on Tuesday, December 3, at 5:00 p.m. in Salon E, Symposium Ca 5.11. The title of his talk is "Strain Relaxation and the Morphology of Thin Films."

\section{MRS Elects Officer, Councillors for 1997}

MRS members elected one officer and three councillors to join 1997 MRS President Robert Hull of the University of Virginia. The annual election ended October 11, 1996.

\section{MRS Officers}

President

Robert Hull (1997)

University of Virginia

Immediate Past President

Carl V. Thompson (1997)

Massachusetts Institute of Technology

Vice President

Robert J. Nemanich (1997)

North Carolina State University

\section{Secretary}

Kevin S. Jones (1997)

University of Florida

Treasurer

* Alan J. Hurd (1998)

Sandia National Laboratories

Xerox Wilson Center

\section{MRS Councillors}

Cammy R. Abernathy (1998)

University of Florida

Harry A. Atwater (1998)

California Institute of Technology

* Theodore M. Besmann (1999)

Oak Ridge National Laboratory

Charles B. Duke (1997)

Ronald Gibala (1997)

University of Michigan

Martin L. Green (1998)

Lucent Technologies/Bell Labs

James M.E. Harper (1997)

IBM T.J. Watson Research Center

Gabrielle G. Long (1997)

National Institute of Standards and Technology
*Amy J. Moll (1999)

Hewlett Packard

Virginia M. Oversby (1998)

VMO Konsult

Manfred Rühle (1997)

Max-Planck-Institut für Metallforschung

*Timothy D. Sands (1999)

University of California-Berkeley

Lyle H. Schwartz (1998)

National Institute of Standards and Technology

Alan I. Taub (1997)

Ford Motor Company

James S. Williams (1998)

Australian National University

(Terms of office expire at the end of the years indicated in parentheses.)

*Newly elected. 